\title{
INTRODUCING COVID-19 AS AN EVOLUTIONARY METABOLIC INFECIOUS DISEASE (EMID)
}

\section{The Prime Cause and Representing Alternative Treatment for COVID-19 (SARS-CoV-2)}

\author{
Sorush Niknamian \\ MD PhD Dr.PH, Military Medicine Department, Liberty University, Virginia, Lynchburg, USA \\ Certified Member of United States Health Professionals (Rank: MAJ Dr.) \\ Email: so.niknamian@gmail.com
}

\section{ABSTRACT}

Background: Coronaviruses are a group of related viruses that cause diseases in mammals and birds. In humans, coronaviruses cause respiratory tract infections that can range from mild to lethal. Mild illnesses include some cases of the common cold, while more lethal varieties can cause SARS, MERS, and COVID-19. The outbreak was identified in Wuhan, China, in December 2019, declared to be a Public Health Emergency of International Concern on 30 January 2020, and recognized as a pandemic on 11 March 2020. Introduction: Coronaviruses are the subfamily Orthocoronavirinae, within the family of Coronaviridae, order Nidovirales, and realm Riboviria. They are enveloped viruses with a positive-sense single-stranded RNA genome and a nucleocapsid of helical symmetry. The genome size of coronaviruses is approximately from 26 to 32 kilobases. Coronaviruses were first discovered in the 1930s and Human coronaviruses were discovered in the 1960s. The earliest ones studied were from human patients with the common cold, which were later named human coronavirus 229E and human coronavirus OC43. Other human coronaviruses have since been identified, including SARS-CoV in 2003, HCoV NL63 in 2004, HKU1 in 2005, MERS-CoV in 2012, and SARS-CoV-2 in 2019. Most of these have involved serious respiratory tract infections. Discussions and Results: Based on our multidisciplinary research, we have found the major cause and some treatments methods for fighting this powerful pathogen. The prime cause of COVID-19 is pushing the mitochondrial to lose MMP. A loss of the MMP by any mechanism leads to functional and structural collapse of the mitochondria and cell death. Mitophagy plays an important role in maintaining mitochondrial homeostasis, but can also eliminate healthy mitochondria in cases such as cell starvation, viral invasion, and erythroid cell differentiation. The mitochondrial fusion and fission are highly dynamic. Viruses specially COVID-19, interfere with these processes to distort mitochondrial dynamic to facilitate their proliferation. Thus, interfering with these processes promotes the interference of different cellular signaling pathways. The severe acute respiratory syndrome coronavirus (SARS-CoV) escapes the innate immune response by translocating its $O R F-9 b$ to mitochondria and promotes proteosomal degradation of dynamin-like protein (Drpl) leading to mitochondrial fission. We also researched on Ultrasonic Energy to destroy the virus which lead to positive results but it needs more future research. The most destructive way of viruses is to enhance Reactive Oxygen Species (ROS) and free radicals in human contaminated cell which cause inflammation in a host cell. ELF-EMF convert free radicals 
into less active molecules and eliminate them into two pathways which has been discussed in the discussion part. Using ELF-EMF affects the second pathway that relies on the activity of the catalase and superoxide dismutase enzymes which is the most effective pathway. For the best result of treatment, is the use of low-frequency magnetic fields (LFMF) plus EMF-ELF which penetrate into deeper tissues, cells and mitochondria. We also have gone through many researches since 1920 and found if we emit the frequency as the same frequency of COVID-19, can cause resonance in the virus and destroy it. So we measured the SARS-CoV-2 frequency by Cyclotron and calculated the frequency of the virus is $30 \mathrm{KHz}-500 \mathrm{KHz}$. Conclusion: COVID-19 (SARS-CoV-2) is one of the most complex virus which has been discovered since 2020. Until today, there has been no Antiviral Drug which can be useful in the treatment of this infectious disease has been discovered till today. COVID-19 genomic sequence containing SARS-CoV, MERS-CoV and Influenza A. Therefore; there is a high possibility of continuing COVID-19 even in summer. To gain the best result in treatment, we should use low-frequency magnetic fields (LFMF) plus EMF which penetrate into deeper tissues, cells and mitochondria in order to reduce ROS and Inflammation. In order to destroy SARS-CoV-2 virus in environment and also in infected individuals, we should use ELF-EMF plus LFMF. We also have gone through many researches since 1920 and found if we emit the frequency as the same frequency of COVID-19, it can cause resonance in the virus and destroy it. So we measured the SARS-CoV-2 frequency by Cyclotron and calculated the frequency of the virus that id is $30 \mathrm{KHz}-500 \mathrm{KHz}$. The differences in the frequencies is due to the size of the virus which is from 26 to 32 Kilobases.

KEYWORDS: COVID-19, Mitochondria, Cyclotron, Resonance, Frequency, MMP, Royal Raymond Rife Protocol, Ultrasound Energy, LFMF, ELF-EMF, Immunodeficiency, Immune Response, Protein Mismatch in COVID-19.

ABBREVIATIONS: DISC: death-inducing signaling complex, ER: endoplasmic reticulum, $\mathrm{HCV}$ : hepatitis $\mathrm{C}$ virus, HBV: hepatitis B virus, PLV: poliovirus, KPSV: Kaposi sarcoma virus, Bid: $\mathrm{BH} 3$ interacting-domain death agonist, WDSV: walleye dermal saecoma virus, HCMV: human cytomegalovirus, EBV: Epstein-Barr virus, SARS-CoV: severe acute respiratory syndrome coronavirus, VDAC: voltage-dependent anion. Channel, HIV: human immunodeficiency virus, IAPs inhibitor of apoptosis proteins, CAVs: Dipolar mode of the confined acoustic vibrations.

\section{INTRODUCTION}

\section{Coronaviruses}

Coronaviruses are a group of related viruses that cause diseases in mammals and birds. In humans, coronaviruses cause respiratory tract infections that can range from mild to lethal. Mild illnesses include some cases of the common cold, while more lethal varieties can cause SARS, MERS, and COVID-19. [1-3] There are yet to be vaccines or antiviral drugs to prevent or treat human coronavirus infections. [2] The 2019-20 coronavirus pandemic is an ongoing pandemic of coronavirus disease 2019 (COVID-19), caused by severe acute respiratory syndrome coronavirus 
2019 (SARS-CoV-2). [4-8] The outbreak of COVID-2019 was identified in Wuhan, China, in December 2019, [8] declared to be a Public Health Emergency of International Concern on 30 January 2020, and recognized as a pandemic on 11 March 2020. [9-10] Coronaviruses are the subfamily Orthocoronavirinae, within the family of Coronaviridae, order Nidovirales, and realm Riboviria. [5-6] They are enveloped viruses with a positive-sense single-stranded RNA genome and a nucleocapsid of helical symmetry. [8] The genome size of coronaviruses is approximately from 26 to 32 kilobases. [7] Coronaviruses were first discovered in the 1930s and Human coronaviruses were discovered in the 1960s. [10] The earliest ones studied were from human patients with the common cold, which were later named human coronavirus $229 \mathrm{E}$ and human coronavirus OC43. [11] Other human coronaviruses have since been identified, including SARSCoV in 2003, HCoV NL63 in 2004, HKU1 in 2005, MERS-CoV in 2012, and SARS-CoV-2 in 2019. Most of these have involved serious respiratory tract infections. [9-11]

\section{Human Body Frequency and Diseases}

Every single particle from non-living small particles to human body (eukaryotic cells and organs) has their own spectacular frequency or vibration Every single cell in human body has repeating vibration or frequency measured in Hz. Higher frequency in the body, results in high immune system and health. normal healthy human body has a frequency of $62-72 \mathrm{MHz}$ When the body drops below this frequency, we the immune system suppresses, human cells sends abnormal signals between Mitochondrion and Nucleus, contamination by viral, bacterial and many diseases occurs [14]. For example, if the normal/healthy human body frequency drops to $58 \mathrm{MHz}$, then the body will likely to get cold, flu and many parasitic like viral and bacterial diseases. The lower the frequency, the higher possibility for infectious diseases. [15]

\section{The Role of Mitochondria as the prime cause of COVID-19}

COVID-19 like Influenza Virus-has 8 RNA segments which can be packaged in different ways when they infect birds, pigs, or humans. [Biology \& Physics at KITP. By Terry Hipsher] Coronaviruses are large pleomorphic spherical particles with bulbous surface projections. [16] The average diameter of the virus particles is around $120 \mathrm{~nm}(.12 \mu \mathrm{m})$. The diameter of the envelope is $\sim 80 \mathrm{~nm}(.08 \mu \mathrm{m})$ and the spikes are $\sim 20 \mathrm{~nm}(.02 \mu \mathrm{m})$ long. The envelope of the virus in electron micrographs appears as a distinct pair of electron dense shells. [17-18] The viral envelope consists of a lipid bilayer where the membrane (M), envelope (E) and spike (S) structural proteins are anchored. [19] Inside the envelope, there is the nucleocapsid, which is formed from multiple copies of the nucleocapsid (N) protein, which are bound to the positive-sense single-stranded RNA genome in a continuous beads-on-a-string type conformation. [20-21] The lipid bilayer envelope, membrane proteins, and nucleocapsid protect the virus when it is outside the host cell. [22] the structure of COVID-19 is so rigid and has very powerful against many antiviral and antimicrobial detergent like alcohol since the outer layer of the virus is lipid and cannot be solved in alcohol based materials.

Based on our calculations, the genome size of COVID-19 as mentioned is from 26 to 32 Kilobases (basepair [bp]). 26 Kilopaepair: 26000 kilobasepair [kb], 0.026 megabasepair [Mb], 0.000026 gigabasepair [Gb], 8666.666667 amino acid [aa], 8666.666667 codon. And 32 Kilobases means: 
32000 kilobasepair [kb], 0.032 megabasepair [Mb], 0.000032 gigabasepair [Gb], 10666.666667 amino acid [aa], 10666.666667 codon. As you observe, the codon and amino-acids of COVID-19 are similar which is very rare in Viruses. When the Codon is similar to amino acid number it is called Codon Mismatch which we observe in COVID-19.

mitochondria are the targets of the reactive oxygen species (ROS) that are produced inside a cell during viral infections, and that mtDNA is a major target of these ROS [23]. Mitochondrial ATP generation requires proteins from the nuclear and mitochondrial genomes. ROS disrupt the oxidative production of ATP, which is required for normal cellular function, because damage of mtDNA disrupts the normal synthesis of proteins needed for mitochondria function and making them suitable targets for attack by ROS produced during infections by viruses and other microorganisms, although ROS also have other cellular targets. In HIV and hepatitis C virus infections, oxidative stress (OS) always plays a dominant pathogenic role. Peterhen and other researchers showed that almost all viruses (DNA/RNA viruses) cause cell death by generating oxidative stress in infected cells [24-26]. The OS generated during chronic hepatitis is associated with hepatic damage, a decrease in reduced glutathione (GSH) and decrease in plasma and hepatic zinc concentration [27-28].

A loss of the MMP leads to imbalances in the membrane potentials of the IM and OM, and then to arrest of normal cellular biosynthetic function and bioenergetics, and finally to a "crisis" within the cell. A loss of the MMP also leads to release of several proapoptotic proteins from the IMS, such as Cyt $\mathrm{C}$ and Smac/DIABLO, as well as caspase independent death effectors, such as apoptosis-inducing factor (AIF) and endonuclease G (EndoG) [9], which have important roles in caspase-independent and caspase-dependent cell death [32]. The MMP transition occurs during the pathogenesis of exogenous factors (e.g., viral proteins, toxins, and prooxidants [33-34]). A prolonged loss of the MMP leads to serious cell damage, from which the cell cannot recover. Therefore, in the intrinsic pathway of apoptosis, any viral factor that influences the MMP has a major impact on cell fate, either by inducing or by blocking cell death [35].

In recent years, there has been an increasing focus on the role of the MMP in disease and health. Thus, several recent models based on in vivo and in vitro studies explain the mechanisms underlying the maintenance and loss of the MMP. A loss of the MMP by any mechanism leads to functional and structural collapse of the mitochondria and cell death [35]. A recent study for first time has shown that dengue virus (DV) infection of human hepatoma cell line (HepG2) leads alteration in the bioenergetic function of mitochondrial morphology leading to MMP loss [28]. The alteration in respiratory properties of HepG2 cells in DV infection results due to decrease in respiratory control ratio (PCR) and $\mathrm{ADP} / \mathrm{O}$ ratio, which suggest significant alteration in mitochondrial morphology. Another additional feature observed by an increase in proton leak termed mitochondrial uncoupling which occurs by leaking of protons through FoF1 ATP synthase from inner membrane into matrix resulting in decrease in MMP loss. Thus, creating an imbalance in ATP synthesis ultimately affects the bioenergetic functions of cell. The biochemical mitochondrial damage induced in cell infected with HCV showed that E1 Protein together with core and NS3 are responsible for ROS production. Core and NS3 induce NO production which causes MMP loss by opening of transition pore [32]. NO could also interact with another free 
radical superoxide (O2-) to form strong peroxynitrite anion (ONOO-), which irreversibly inhibits multiple respiratory complexes (complexes I, II and IV) and aconitase, and activate proton leak and permeability transition pore [30,31]. Therefore, interfering with energy metabolism by disrupting the ATP synthesis of cell results in modulation of mitochondrial function.

\section{Effects of viral infections on mitochondrial processes}

Mitochondria undergo a number of processes, such as fusion and fission, in normally functioning healthy cells. However, when mitochondria develop abnormalities, the cell can destroy it by the process of mitophagy [32]. Cells typically eliminate unhealthy mitochondria by mitochondrial fission; they typically use mitochondrial fusion to recycle matrix metabolites, including mtDNA and mitochondrial membranes, for the assembly of new and healthy mitochondria. Therefore, these three processes mitophagy, fission, and fusion are interlinked, and they all play prominent roles in maintaining healthy cells [33]. Mitophagy plays an important role in maintaining mitochondrial homeostasis, but can also eliminate healthy mitochondria in cases such as cell starvation, viral invasion, and erythroid cell differentiation [34-35]. The mitochondrial fusion and fission are highly dynamic. Viruses interfere with these processes to distort mitochondrial dynamic to facilitate their proliferation. Thus, interfering with these processes promotes the interference of different cellular signaling pathways [36-38]. New castal virus (NDV) uses strategy that interferes with P62mediated mitophagy to promote viral propagation [Castanier $\mathrm{C}$ et al.2010]. The severe acute respiratory syndrome coronavirus (SARS-CoV) escapes the innate immune response by translocating its ORF-9b to mitochondria and promotes proteosomal degradation of dynamin-like protein (Drp1) leading to mitochondrial fission [Shi C-S et al,.2014]. However, still more studies are needed to explain the exact role of mitophagy in the viral disease pathogenesis, which regulates the cell death process [38]. For the best results of treatment with EMFFoletti A. Lisi A. Ledda M. et al. at 2009 demonstrated that biological effects of low-frequency magnetic fields may penetrate into deeper tissues [42].

\section{DISCUSSIONS}

The main aim of this research is to obtain the most possible positive biophysical treatment method instead of viral vaccination against COVID-19 (SARS-CoV-2) and introducing The Role of Mitochondria as the prime cause of COVID-19 which has been ignored in causing this pandemic fatal disease. Also we aim to predict the future of new virus after the pandemic of COVID-19.

As discussed before in the introduction session, the most destructive way of viruses is to reduce Reactive Oxygen Species and free radicals in human contaminated cell which cause inflammation in a host cell. ELF-EMF changes the free radical levels in the organism. Free radicals in the body are eliminated through two pathways. The first pathway is the non-enzymatic pathway including glutathione, vitamins, carotenoids and flavonoids, while the second pathway relies on the activity of the enzyme, which is the most effective pathway. The key enzymes include catalase and superoxide dismutase. ELF-EMF convert free radicals into less active molecules and eliminate them [38-39]. Foletti A. Lisi A. Ledda M. et al. at 2009 showed biological effects of low-frequency magnetic fields may penetrate into deeper tissues. Therefore; in order to gain the best results, is to 
use Low Frequency Magnetic Fields (LFMF) which had been tested and demonstrated by Royal Raymond Rife in 1920. [42] There are several studies shoes LFMF benefits in killing viruses as well as enhancing the immune system functions. [43-50] Giladi et al. showed EMF of intermediate frequency was effective in arresting the growth of cells. Kirson et al. demonstrated that this direct inhibitory effect on cell growth can be used for therapeutic purposes in the treatment of viral infections including Virions. [51-52].

G. SCHERBA et al. used Ultrasonic Energy on feline herpesvirus type 1 and feline calicivirus which infects cat's respiratory organ they used $26 \mathrm{kHz}$ of $-212.3 \mathrm{~dB}$ (re: $1 \mathrm{~V} /, \mathrm{uPa}$ ). Their research reached positive results on the treatment of these viruses.

In 1980s, Robach et al. [56] and Cerf [57] demonstrated that ultrasonic energy can be absorbed by viruses and terminated them when use appropriately. This means viruses are sensitive to Ultrasonic energy.

In 2000, Babincová et al. [58] hypothesized that viruses can be inactivated by generating the corresponding resonance ultrasound vibrations of viruses, which is in the GHz region. Based on this hypothesis. Dykeman, E. C. et al., started investigating the vibrational modes of viruses in this frequency range [59-62]. Dykeman, E. C. et al. (did two major researches), Tsen K. T. et aland Liu T. M. did the same research and reached positive results.

Dipolar mode of the confined acoustic vibrations (CAVs) inside viruses can be resonantly excited by microwaves of the same frequency with a resonant microwave absorption effect [63]. The observed microwave resonance absorption phenomenon indicates a possible structure-resonant energy transfer (SRET) effect from electromagnetic waves (EM waves) to CAVs of viruses. Theoretically this SRET process is an efficient way to excite the vibrational mode of the whole virus structure due to a $100 \%$ energy conversion of a photon into a phonon of the same frequency, but the overall SRET efficiency is also related to the mechanical properties of the surrounding environment [64], which influences the quality factor of the oscillator (virus). A study on the SRET efficiency to inactive virus is thus highly desired and it will determine if this SRET phenomenon provides a solution to inactivate airborne viruses in open public for epidemic prevention. [Szu-Chi Yang et al. 2015]

Szu-Chi Yang et. Al applied microwave frequency of $7 \mathrm{GHz}$ in many airborne viruses, the applied average microwave power density was $308 \mathrm{~W} / \mathrm{m} 2$, corresponding to an effective electric field of $167 \mathrm{~V} / \mathrm{m}$ inside the specimens. They also showed that the frequency of $7 \mathrm{GHz}$, can cause resonance in all examined viruses, SARS-CoV-1, MERS-CoV and many types of viruses including influenza A virus. They also concluded that structure-resonant energy transfer effect from microwaves to virus can be efficient enough so that airborne viruses were inactivated with reasonable microwave power density is safe for the open public. [Szu-Chi Yang et al. 2015] Their data demonstrates that all the viruses discussed in Szu-Chi Yang experiment has Virion structure and possibly can be experiment in reducing the pandemic of SARS-CoV-2 and even inactivate and cause resonance which yields in the destroying the structure of the COVID-19.

The first large scale application of ultrasound was around World War II. Sonar systems were being built and used to navigate submarines. It was realized that the high intensity ultrasound waves that 
they were using were heating and killing fish. This led to research in tissue heating and healing effects. Since the 1940s, ultrasound has been used by physical and occupational therapists for therapeutic effects. [53] Unfortunately, there are many studies and researches has been done on Microbial Infections, hence; there has been small researches in the field of Viral Infection prevention and treatments, specially Virions like SARS-CoV-1, MERS and Influenza viruses which attack respiratory organs and has not been any antiviral vaccines introduced since these viruses mutates very quickly and adaptable to the environment and their hosts.

In conclusion, both low-frequency magnetic fields and Ultrasonic Energy Therapy were useful against many Viral and mostly on Bacterial diseases.

\section{Calculating natural frequency of SARS-CoV-2}

The neglected aspect of killing Virions including SARS-CoV-2 is causing resonance in viruses. Viruses only becomes powerful inside the body of the host and outside the living hosts they are like nonliving particles. Let's assume COVID-19 as a mechanical system or small particle like a ball. The natural frequency of a simple mechanical system consisting of a weight suspended by a spring is: The natural frequency of a simple mechanical system consisting of a weight suspended

by a spring is: $f=\frac{1}{2 \pi} \sqrt{\frac{k}{m}}$

A swing set is a simple example of a resonant system with which most people have practical experience. It is a form of pendulum. If the system is excited (pushed) with a period between pushes equal to the inverse of the pendulum's natural frequency, the swing will swing higher and higher, but if excited at a different frequency, it will be difficult to move. The resonance frequency of a pendulum, the only frequency at which it will vibrate, is given approximately, for small displacements, by the equation:

$f=\frac{1}{2 \pi} \sqrt{\frac{g}{L}}$

where $\mathrm{g}$ is the acceleration due to gravity (about $9.8 \mathrm{~m} / \mathrm{s} 2$ near the surface of Earth), and $\mathrm{L}$ is the length from the pivot point to the center of mass. (An elliptic integral yields a description for any displacement). Note that, in this approximation, the frequency does not depend on mass. Mechanical resonators work by transferring energy repeatedly from kinetic to potential form and back again. In the pendulum, for example, all the energy is stored as gravitational energy (a form of potential energy) when the bob is instantaneously motionless at the top of its swing. This energy is proportional to both the mass of the bob and its height above the lowest point. As the bob descends and picks up speed, its potential energy is gradually converted to kinetic energy (energy of movement), which is proportional to the bob's mass and to the square of its speed. When the bob is at the bottom of its travel, it has maximum kinetic energy and minimum potential energy. The same process then happens in reverse as the bob climbs towards the top of its swing. Some resonant objects have more than one resonance frequency, particularly at harmonics (multiples) of the strongest resonance. It will vibrate easily at those frequencies, and less so at other frequencies. 
[54-55] We have to mention that calculating the resonance of the virus with this method is not possible since we have to produce a spring in $\mathrm{nm}$ to calculate the resonance of event giant viruses.

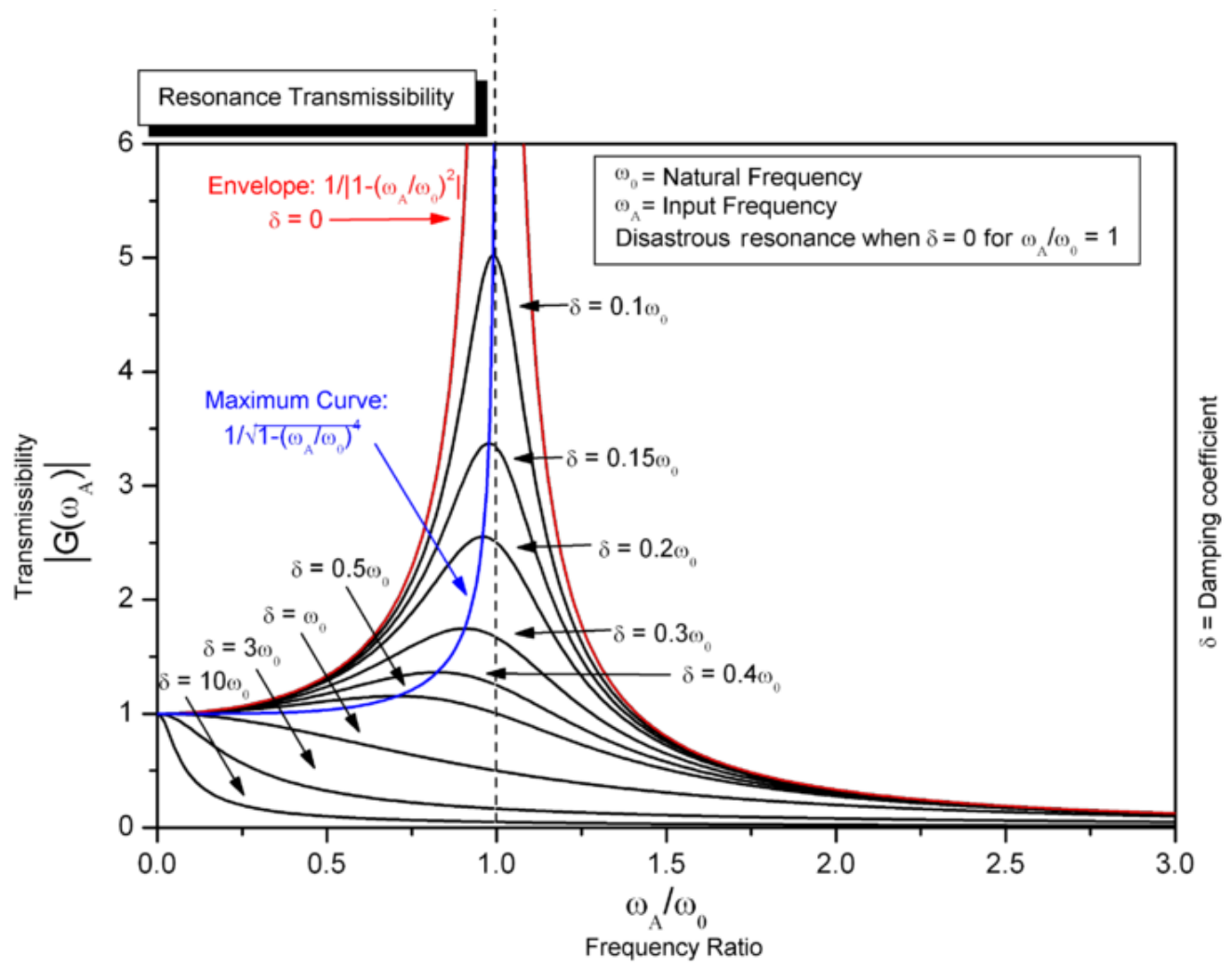

Picture (1): Example of increase of amplitude as damping decreases and frequency approaches resonant frequency of a driven damped simple harmonic oscillator [54-55]

Based on research by JM Kim in 2020, particle size (COVID-19) ranged from 70-90 nm. [JeongMin Kim, et al. 2020]. Therefore; the radius of the virus is between $35-45 \mathrm{~nm}$.

Since the virus as spherical, we can calculate the volume, approximate surface and ....

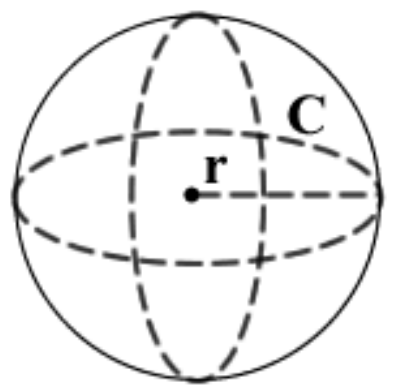

Picture (2): Spherical shape. $\mathrm{r}=$ radius, $\mathrm{V}=$ volume, $\mathrm{A}=$ surface area, $\mathrm{C}=$ circumference, $\pi=\mathrm{pi}=3.1415926535898, \sqrt{ }=$ square root.

As we know the genome size of coronaviruses ranges from approximately 26 to 32 kilobases. Therefore: 
(1) To gain the resonance frequency of the virus, we did the following calculations:

Radius: $\mathrm{r}=35 \mathrm{~mm}=3.5 \mathrm{e}-5 \mathrm{~nm}$, volume: $\mathrm{V}=1.8 \times 10^{-13} \mathrm{~mm} 3$, surface area: $\mathrm{A}=1.54 \times 10^{-8} \mathrm{~mm} 2$ Therefore, due to $M=4 / 3 \cdot \pi \cdot \mathrm{r}^{3} \cdot \mathrm{mD}$ formula, the mass of virus is: $4 / 3 . \pi \cdot 35^{3}=1.7959438 \mathrm{e}-26$ Nomogram. Therefore; the energy of our system based on the calculations above for COVID-19 with Radius of 35-45 nm equals: $30 \mathrm{KHz}-500 \mathrm{KHz}$. This is the approximate resonance frequency of the virus.

(2) In order to gain the Codon and the number of Amino-Acids of Coronavirus we did the following calculations:

Radius: $45 \mathrm{~mm}=4.5 \mathrm{e}-7 \mathrm{~nm}$, Volume: $\mathrm{V} \approx 3.82 \times 10^{-23} \mathrm{~mm} 3$, Surface area: $\mathrm{A} \approx 2.54 \times 10^{-12}$. Therefore, due to $M=4 / 3 \cdot \pi \cdot r^{3} \cdot \mathrm{mD}$ formula, the mass of virus is: $3.8170351 \mathrm{e}-26$.

The genome size of coronaviruses including COVID-19 ranges from approximately 26 to 32 kilobases. In 26 bp: amino acid [aa]: 8.666667 and codon: 8.666667. in 32 bp we have: amino acid [aa]: 10.666667 and codon: 10.666667 . 
As we observe, Coronaviruses number of amino-acids are equals their codons. Which means $\mathrm{Ka} / \mathrm{Ks}$ ratio (also known as $\omega$ or $\mathrm{dN} / \mathrm{dS}$ ratio) equals 1 (One). this result proves that Coronaviruses including COVID-19 use Neutral Selection along with Natural Selection introduced by Charles Darwin and Lamarckian Evolution. [65]

We have measured the frequency of COVID-19 by Cyclotron (Synchrocyclotron). Therefore; the result is: $30 \mathrm{KHz}-500 \mathrm{KHz}$ which has no side effects in human cells since The most restrictive limits on whole-body exposure are in the frequency range of 30-300 $\mathrm{MHz}$ where the human body absorbs RF energy most efficiently when the whole body is exposed. [70]

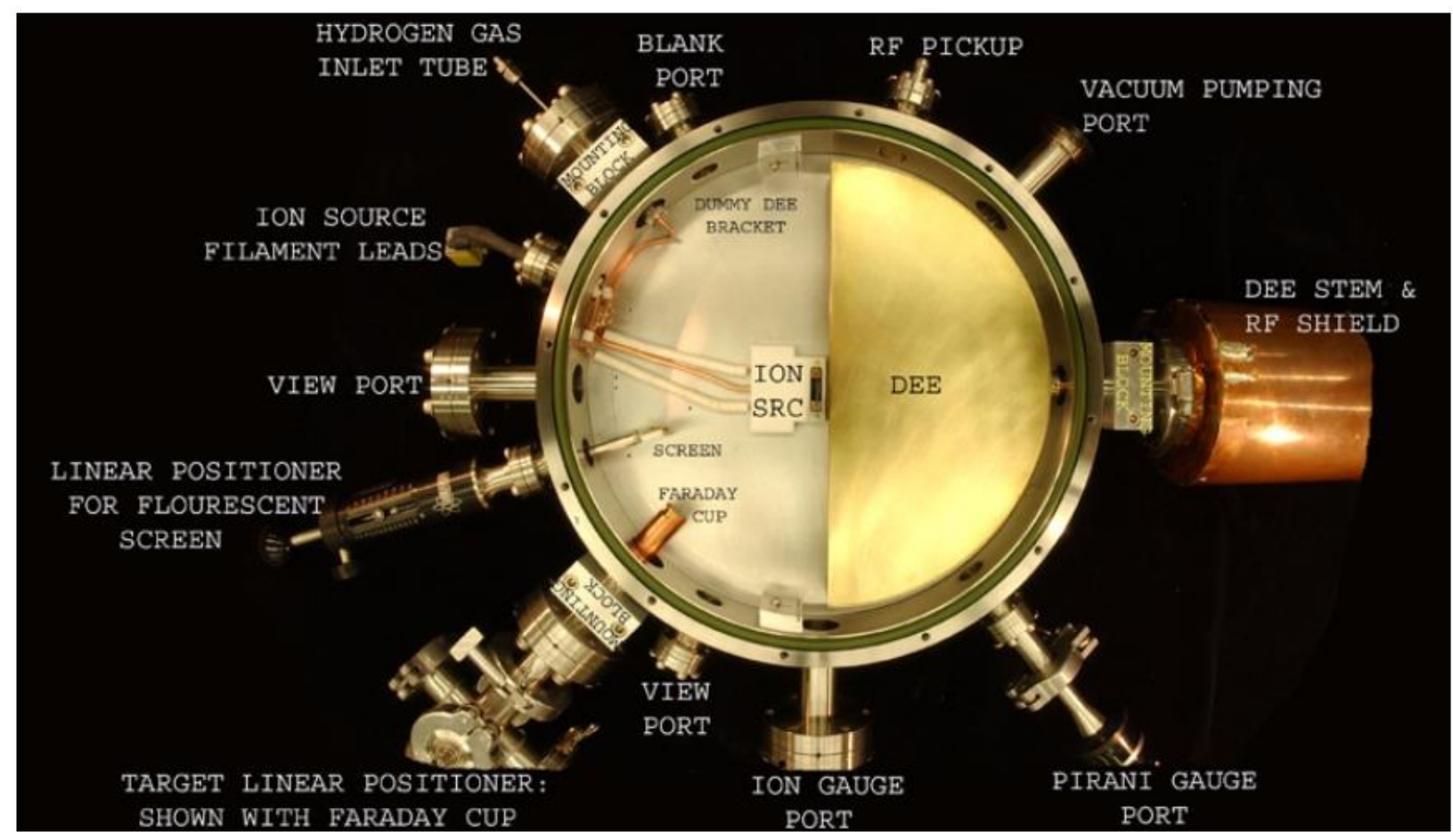

Picture (3): cyclotron is a kind of particle accelerator (a machine which uses electromagnetic fields for propagation of charged particles to approx. light speed and contain them in a welldefined beam) in which the charged particles get accelerated in an outward direction from the center, following a spiral path. [70]

\section{THE IMPACT OF HUMAN BODY FREQUECY ON HEALTH}

The total number of human cells at anywhere is from 15 trillion to 724 trillion [D. C. Savage. 1977]. As mentioned before, normal healthy human body has a frequency of $62-72 \mathrm{MHz}$ Therefore to find the normal frequency of each living human cell we reach;

$62 \mathrm{MHz} / 15$ Trillion $=4.13333333 \times 10^{-6}$ hertz (Approximately $4.1 \times 10^{-6}$ hertz), $72 \mathrm{MHz} / 15$ Trillion $=4.8 \times 10^{-6}$ hertz, $62 \mathrm{MHz} / 724$ Trillion $=8.56353591 \times 10^{-8}$ hertz $($ Approximately $8.5 \times$ $10^{-8}$ hertz), $72 \mathrm{MHz} / 724$ Trillion $=9.94475138 \times 10^{-8}$ hertz (Approximately $10 \times 10^{-8}$ hertz. [6667] 


\section{RESULTS}

Based on our research, COVID-19 (SARS-CoV-2, 2019-Coronavirus) genome/size is between 2632 Kilobases. with our approximate mathematical calculations, the virus volume is between $1.8 \times 10-13 \mathrm{~mm} 3-\mathrm{V} \approx 3.82 \times 10-23$.

Based on Jeong-Min Kim et al [68]., the virus genome sequence includes SARS-CoV-2, SARS$\mathrm{CoV}$, and MERS-CoV which shows the virus used genetic drift (Sewall Wright effect) with help of natural selection to become more vital, adaptable to the environment including human/mammalian/Birds host. MERS-CoV (Middle East respiratory syndrome) can tolerate high temperature environment because these mammal's habitat is deserts. [69] Therefore we cannot forecast the pandemic ends with higher temperature of the environment (Due to this result and also Lamarckian Evolution)

We have also measured the frequency of COVID-19 by Cyclotron and calculated the resonance frequency of COVID-19. the result is: $30 \mathrm{KHz}-500 \mathrm{KHz}$ which has not negative effects on human health and cells or organs.

\section{CONCLUSION}

In this research, we have gone through most viral infection treatment through history and also did some researches on finding the magnetic field of the virus by Cyclotron. There had found no evidence supporting positive impact of coronavirus and Influenza contamination vaccinations until today which really heal the patient forever in his lifetime. The major cause of COVID-19 is loss of the MMP in mitochondria which leads to imbalances in the membrane potentials of the IM and $\mathrm{OM}$, and then to arrest of normal cellular biosynthetic function and bioenergetics, and finally called crisis within the cell. Therefore, in the intrinsic pathway of apoptosis, any viral factor that influences the MMP has a major impact on cell fate, either by inducing or by blocking cell death. Also we have observed that the number of Amino Acids and Capsids in COVID-19 are the same which is called Protein Mismatch which is influential. However; there are some treatments have been mentioned in this article. Ultrasound Energy which have been used for decades to destroy mainly bacteria, but have been useful in destroying many viruses as well. We in introduced ELFEMF that convert free radicals into less active molecules and eliminate them into two pathways which has been discussed in the discussion part. Using ELF-EMF affects the second pathway that relies on the activity of the catalase and superoxide dismutase enzymes which is the most effective pathway. To gain the best result in treatment, we should use low-frequency magnetic fields (LFMF) plus EMF which penetrate into deeper tissues, cells and mitochondria in order to reduce ROS and Inflammation. In order to destroy SARS-CoV-2 virus in environment and also in infected individuals, we should use ELF-EMF plus LFMF. We also have gone through many researches since 1920 and found if we emit the frequency as the same frequency of COVID-19, it can cause resonance in the virus and destroy it. So we measured the SARS-CoV-2 frequency by Cyclotron and calculated the frequency of the virus that id is $30 \mathrm{KHz}-500 \mathrm{KHz}$. The differences in the 
frequencies is due to the size of the virus which is from 26 to 32 Kilobases. Based on recent research in Korea, COVID-19 genomic sequence containing SARS-CoV, MERS-CoV and Influenza A. Therefore; there is a possibility of the pandemic be continued even in summer.

\section{REFERENCES}

[1] "Virus Taxonomy: 2018b Release". International Committee on Taxonomy of Viruses (ICTV). March 2019. Archived from the original on 2018-03-04. Retrieved 2020-01-24.

[2] "2017.012-015S" (xlsx). International Committee on Taxonomy of Viruses (ICTV). October 2018. Archived from the original on 2019-05-14. Retrieved 2020-01-24^

[3] "ICTV Taxonomy history: Orthocoronavirinae". International Committee on Taxonomy of Viruses (ICTV). Retrieved 2020-01-24.

[4] Fan Y, Zhao K, Shi ZL, Zhou P (March 2019). "Bat Coronaviruses in China". Viruses. 11 (3): 210. doi:10.3390/v11030210. PMC 6466186. PMID 30832341.

[5] Groot RJ, Baker SC, Baric R, Enjuanes L, Gorbalenya AE, Holmes KV, Perlman S, Poon L, Rottier PJ, Talbot PJ, Woo PC, Ziebuhr J (2011). "Family Coronaviridae". In King AM, Lefkowitz E, Adams MJ, Carstens EB, International Committee on Taxonomy of Viruses, International Union of Microbiological Societies. Virology Division (eds.). Ninth Report of the International Committee on Taxonomy of Viruses. Oxford: Elsevier. pp. 806-28. ISBN 978-0-12-384684-6.

[6] International Committee on Taxonomy of Viruses (2010-08-24). "ICTV Master Species List 2009- $110 "$ (xls).

[7] Woo, Patrick C. Y.; Huang, Yi; Lau, Susanna K. P.; Yuen, Kwok-Yung (2010-08-24). "Coronavirus Genomics and Bioinformatics Analysis". Viruses. 2 (8): 1804-1820. doi:10.3390/v2081803. ISSN 1999-4915. PMC 3185738. PMID 21994708. Coronaviruses possess the largest genomes [26.4 kb (ThCoV HKU12) to $31.7 \mathrm{~kb}$ (SW1)] among all known RNA viruses (Figure 1) $[2,13,16]$.

[8] Almeida JD, Berry DM, Cunningham CH, Hamre D, Hofstad MS, Mallucci L, McIntosh K, Tyrrell DA (November 1968). "Virology: Coronaviruses". Nature. 220 (5168): 650. doi:10.1038/220650b0. PMC 7086490. [T] here is also a characteristic "fringe" of projections 200 A long, which are rounded or petal shaped ... This appearance, recalling the solar corona, is shared by mouse hepatitis virus and several viruses recently recovered from man, namely strain B814, $229 \mathrm{E}$ and several others.

[9] Lau, Hien; Khosrawipour, Veria; Kocbach, Piotr; Mikolajczyk, Agata; Ichii, Hirohito; Schubert, Justyna; Bania, Jacek; Khosrawipour, Tanja (March 2020). "Internationally lost COVID-19 cases". Journal of Microbiology, Immunology and Infection. doi: 10.1016/j.jmii.2020.03.013. PMID 32205091. The total number of cases may not necessarily add up due to the frequency of values updating for each individual location. ${ }^{\wedge}$ In summary, this article 
is about the coronavirus pandemic, which is caused by the disease COVID-19, which is caused by the virus SARS-CoV-2.

[10] WHO-China Joint Mission (16-24 February 2020). "Report of the WHO-China Joint Mission on Coronavirus Disease 2019 (COVID-19)" (PDF). World Health Organization. Retrieved 8 March 2020.

[11] Cyranoski D (26 February 2020). "Mystery deepens over animal source of coronavirus". Nature. 579 (7797): 18-19. Bibcode:2020Natur.579...18C. doi:10.1038/d41586-020-00548-w. PMID 32127703.

[12] Preslar D, Borger J. Body Piercing Infections. [Updated 2019 Nov 8]. In: StatPearls [Internet]. Treasure Island (FL): StatPearls Publishing; 2020 Jan-. Available from: https://www.ncbi.nlm.nih.gov/books/NBK537336/.

[13] Grant SS, Hung DT. Persistent bacterial infections, antibiotic tolerance, and the oxidative stress response. Virulence. 2013;4(4):273-283. doi:10.4161/viru.23987.

[14] Desert Gate the Rife Handbook of Frequency Therapy and Holistic Health by Nenah Sylver PhD (2011-05-03). ISBN 10: 0981807518 / ISBN 13: 9780981807515. Published by Desert Gate, 2009.

[15] Lawrence R. Freedman. 2012. Current Topics in Infectious Disease: Infective Endocarditis and Other Intravascular Infections.

[16] Dr. Royal R. Rife. A Rare Recording of Dr. Royal R. Rife. Release Date: July 20, 2018. Publisher: Listen \& Live Audio. Unabridged Audiobook.

[17] Goldsmith CS, Tatti KM, Ksiazek TG, Rollin PE, Comer JA, Lee WW, et al. (February 2004). "Ultrastructural characterization of SARS coronavirus". Emerging Infectious Diseases. 10 (2): 320-26. doi:10.3201/eid1002.030913. PMC 3322934. PMID 15030705. Virions acquired an envelope by budding into the cisternae and formed mostly spherical, sometimes pleomorphic, particles that averaged $78 \mathrm{~nm}$ in diameter (Figure 1A).

[18] Neuman BW, Adair BD, Yoshioka C, Quispe JD, Orca G, Kuhn P, et al. (August 2006). "Supramolecular architecture of severe acute respiratory syndrome coronavirus revealed by electron cryomicroscopy". Journal of Virology. 80 (16): 7918-28. doi:10.1128/JVI.00645-06. PMC 1563832. PMID 16873249. Particle diameters ranged from 50 to $150 \mathrm{~nm}$, excluding the spikes, with mean particle diameters of 82 to $94 \mathrm{~nm}$; Also See Figure 1 for double shell.

[19] Fehr AR, Perlman S (2015). Maier HJ, Bickerton E, Britton P (eds.). "Coronaviruses: an overview of their replication and pathogenesis". Methods in Molecular Biology. Springer. 1282: 1-23. doi:10.1007/978-1-4939-2438-7_1. ISBN 978-1-4939-2438-7. PMC 4369385. PMID 25720466. See section: Virion Structure.

[20 Lai MM, Cavanagh D (1997). "The molecular biology of coronaviruses". Advances in Virus Research. 48: 1-100. doi:10.1016/S0065-3527(08)60286-9. ISBN 9780120398485. PMID 9233431. 
[21] Chang CK, Hou MH, Chang CF, Hsiao CD, Huang TH (March 2014). "The SARS coronavirus nucleocapsid protein-forms and functions". Antiviral Research. 103: 39-50. doi:10.1016/j.antiviral.2013.12.009. PMID 24418573. See Figure 4c.

[22] Neuman BW, Kiss G, Kunding AH, Bhella D, Baksh MF, Connelly S, et al. (April 2011). "A structural analysis of M protein in coronavirus assembly and morphology". Journal of Structural Biology. 174 (1): 11-22. doi: 10.1016/j.jsb.2010.11.021. PMC 4486061. PMID 21130884. See Figure 10.

[23] Reshi L, Wang HV, Hui CF, Su YC, Hong JR. Anti-apoptotic genes Bcl-2 and Bcl-xL overexpression can block iridovirus serine/threonine kinase-induced Bax/mitochondria-mediated cell death in GF-1 cells. Fish \& Shellfish Immunology. 2017; 61:120-129.

[24] Peterhans E, Grob M, Urge TB, Zanoni R. Virus-induced formation of reactive oxygen intermediates in phagocytic cells. Free Radical Research Communications. 1987;3(1-5):39-46.

[25] Vierucci A, DeMartino M, Graziani E. A mechanism for liver cell injury in viral hepatitis: Effects of hepatitis B virus on neutrophil function in vitro and in children with chronic active hepatitis. Pediatric Research. 1983;17(10):814-820.

[26] Muller F. Reactive oxygen intermediates and human immunodeficiency virus (HIV) infection. Free Radical Biology and Medicine. 1992;13(6):651-657.

[27] Boya P, Peña AD, Beloquietal O. Antioxidant status and glutathione metabolism in peripheral blood mononuclear cells from patients with chronic hepatitis C. Journal of Hepatology. 1999;31(5):808-814.

[28] Bianchi GP, Marchesini G, Brizietal M. Nutritional effects of oral zinc supplementation in cirrhosis. Nutrition Research. 2000;20(8):1079-1089.

[29] Reshi L, Su YC, Hong JR. RNA viruses: ROS-mediated cell death. International Journal of Cell Biology. 2014;2014:467-452.

[30] Reshi L, Wu JL, Wang HV, Hong JR. Aquatic viruses induce host cell death pathways and its application. Virus Research. 2016; 211:133-144.

[31] Reshi L, Hong JR. Mitochondria as a favorite organelle for invading viruses. Molecular Biology. 2017;6:181.

[32] Reshi L, Su YC, Hong JR. RNA viruses: ROS-mediated cell death. International Journal of Cell Biology. 2014;2014:467-452.

[33] Machida $\mathrm{K}$ et al. Hepatitis $\mathrm{C}$ virus infection activates the immunologic (type II) isoform of nitric oxide synthase and thereby enhances DNA damage and mutations of cellular genes. Journal of Virology. 2004;78(16):8835-8843.

[34] Reshi L, Wu JL, Wang HV, Hong JR. Aquatic viruses induce host cell death pathways and its application. Virus Research. 2016;211:133-144. 
[35] Machida $\mathrm{K}$ et al. Hepatitis $\mathrm{C}$ virus infection activates the immunologic (type II) isoform of nitric oxide synthase and thereby enhances DNA damage and mutations of cellular genes. Journal of Virology. 2004;78(16):8835-8843.

[36] Reshi L, Hong JR. Mitochondria as a favourite organelle for invading viruses. Molecular Biology. 2017;6:181.

[37] Meng G, Xia M, Wang D, Chen A, Wang Y, Wang H, Yu D, Wei J. Mitophagy promotes replication of oncolytic Newcastle disease virus by blocking intrinsic apoptosis in lung cancer cells. Oncotarget. 2014;5(15):6365-6374.

[38] Xia M, Meng G, Jiang A, et al. Mitophagy switches cell death from apoptosis to necrosis in NSCLC cells treated with oncolytic measles virus. Oncotarget. 2014;5(11):3907.

[39] Valko M. Leibfrietz D. Moncol J. Cronin M. T. D. Mazur M. Tesler J. 2007, Cronin MTD., Mazur M., Tesler J. 2007. Free radicals and antioxidants in normal physiological functions and human disease. The international Journal of Biochemistry \& Cell Biology39 4484.

[40] Moore K. Roberts J. L. 1998 Measurement of lipid peroxidation. Free Radical Research 28 659671.

[41] Foletti A. Lisi A. Ledda M. et al. 2009 Cellular ELF signals as a possible tool in informative medicine. Electromagnetic Biology and Medicine. 28 (1), 71 EOF 79 EOF -79.

[42] Barry Lynes. Rife's World of Electromedicine: The Story, the Corruption and the Promise. February 12, 2009.

[43] Guerriero F, Ricevuti G. Extremely low frequency electromagnetic fields stimulation modulates autoimmunity and immune responses: a possible immuno-modulatory therapeutic effect in neurodegenerative diseases. Neural Regen Res. 2016;11(12):1888-1895. doi:10.4103/1673-5374.195277.

[44] Patruno A1, Tabrez S2, Pesce M1, Shakil S3, Kamal MA2, Reale M4. Effects of extremely low frequency electromagnetic field (ELF-EMF) on catalase, cytochrome P450 and nitric oxide synthase in erythro-leukemic cells. Life Sci. 2015 Jan 15;121:117-23. doi: 10.1016/j.lfs.2014.12.003. Epub 2014 Dec 11.

[45] Simkó M1, Mattsson MO. Extremely low frequency electromagnetic fields as effectors of cellular responses in vitro: possible immune cell activation. J Cell Biochem. 2004 Sep 1;93(1):8392.

[46] Komaki A1, Khalili A2, Salehi I2, Shahidi S2, Sarihi A2, Effects of exposure to an extremely low frequency electromagnetic field on hippocampal long-term potentiation in rat. Brain Res. 2014 May 20;1564:1-8. doi: 10.1016/j.brainres.2014.03.041. Epub 2014 Apr 12.

[46] Belyaev I, Dean A, Eger H, Hubmann G, Jandrisovits R, Kern M, Kundi M, Moshammer H, Lercher P, Müller K, Oberfeld G, Ohnsorge P, Pelzmann P, Scheingraber C, Thill R. EUROPAEM EMF Guideline 2016 for the prevention, diagnosis and treatment of EMF-related health problems and illnesses. Rev Environ Health. 2016 Sep 1;31(3):363-97. doi: 10.1515/reveh-2016-0011. 
[47] Zhang Y1, Liu X, Zhang J, Li N. Short-term effects of extremely low frequency electromagnetic fields exposure on Alzheimer's disease in rats. Int J Radiat Biol. 2015 Jan;91(1):28-34. doi: 10.3109/09553002.2014.954058. Epub 2014 Nov 14.

[48] Akbarnejad Z1,2, Esmaeilpour K1, Shabani M1, Asadi-Shekaari M1, Saeedi Goraghani M1, Ahmadi-Zeidabadi M1. Spatial memory recovery in Alzheimer's rat model by electromagnetic field exposure. Int J Neurosci. 2018 Aug;128(8):691-696. doi: 10.1080/00207454.2017.1411353. Epub 2017 Dec 20.

[49] Mahaki H1,2, Tanzadehpanah H2, Jabarivasal N3, Sardanian K1,2, Zamani A1,4. A review on the effects of extremely low frequency electromagnetic field (ELF-EMF) on cytokines of innate and adaptive immunity. Electromagn Biol Med. 2019;38(1):84-95. doi: 10.1080/15368378.2018.1545668. Epub 2018 Dec 6.

[50] Simkó M1, Mattsson MO. Extremely low frequency electromagnetic fields as effectors of cellular responses in vitro: possible immune cell activation. J Cell Biochem. 2004 Sep 1;93(1):8392.

[51] Kirson E. Schneiderman R. Dbaly V. Tovarys F. Et al. 2009 Chemotherapeutic treatment efficacy and sensitivity are increased by adjuvant alternating electric fields. BMC Medical Physics 91

[52] Banik S. Bandyopadhyay S. Ganguly S. 2003 Bioeffects of microwave-a brief review. Bioresource Technology. 87(2), 155 EOF 9 EOF.

[53] ^ Woo, Joseph. "A short History of the development of Ultrasound in Obstetrics and Gynecology". esource Discovery Network, University of Oxford. Retrieved March 12, 2012.

[54] K. Billah and R. Scanlan (1991), Resonance, Tacoma Narrows Bridge Failure, and Undergraduate Physics Textbooks, American Journal of Physics, 59(2), 118-124 (PDF)

[55] Allensworth, et al., United States Patent 4,524,295. June 18, 1985.

[56] Robach, Y. et al. Ultrasonic absorption evidence for structural fluctuations in frog virus 3 and its subparticles. Proc. Nat. Acad. Sci. 80, 3981 (1983).

[57] 2. Cerf, R. Absolute measurement of enhanced fluctuations in assemblies of biomolecules by ultrasonic techniques. Biophys J. 47, 751 (1985).

[58] Babincová, M., Sourivong, P. \& Babinec, P. Resonant absorption of ultrasound energy as a method of HIV destruction. Med. Hypotheses 55, 450 (2000).

[59] Dykeman, E. C. \& Sankey, O. F. Low Frequency Mechanical Modes of Viral Capsids: An Atomistic Approach. Phys. Rev. Lett. 100, 028101 (2008). 5. Balandin, A. A. \& Fonoberov, V. A. [60] Vibrational modes of nano-template viruses. J. Biomed. Nanotechnol. 1, 90 (2005).

[61] Dykeman, E. C. \& Sankey, O. F. Atomistic modeling of the low-frequency mechanical modes and Raman spectra of icosahedral virus capsids. Phys. Rev. E. 81, 021918 (2010). 
[62] Tsen, K. T. et al. Raman scattering studies of the low-frequency vibrational modes of bacteriophage M13 in water-observation of an axial torsion mode. Nanotechnology 17, 5474 (2006).

[63] Liu, T.-M. et al. Microwave resonant absorption of viruses through dipolar coupling with confined acoustic vibrations. Appl. Phys. Lett. 94, 043902 (2009).

[64] Liu, T.-M. et al. Effects of hydration levels on the bandwidth of microwave resonant absorption induced by confined acoustic vibrations. Appl. Phys. Lett. 95, 173702 (2009).

[65] Rocha, E. P. C.; Smith, J. M.; Hurst, L. D.; Holden, M. T. G.; Cooper, J. E.; Smith, N. H.; Feil, E. J. (2006). "Comparisons of $\mathrm{dN} / \mathrm{dS}$ are time dependent for closely related bacterial genomes". Journal of Theoretical Biology. 239 (2): 226-35. doi: 10.1016/j.jtbi.2005.08.037. PMID 16239014.

[66] Ray Abel. Frequency: Harness the power of human Frequency and change your life forever, 2016.

[67] Penney Peirce, Frequency: The Power of Personal Vibration, 2011.

[68] Jeong-Min Kim a, Yoon-Seok Chung a, Hye Jun Jo a, Nam-Joo Lee a, Mi Seon Kim a, Sang Hee Woo a, Sehee Park a, Jee Woong Kim b, Heui Man Kim a, Myung-Guk Han a, *, Identification of Coronavirus Isolated from a Patient in Korea with COVID-19 Osong Public Health Res Perspect 2020;11(1):3-7, https://doi.org/10.24171/j.phrp.2020.11.1.02. 2020.

[69] https://www.fcc.gov/engineering-technology/electromagnetic-compatibility-division/radiofrequency-safety/faq/rf-safety.

[70] Hemida MG, Perera RA, Wang P, Alhammadi MA, Siu LY, Li M, Poon LL, Saif L, Alnaeem A, Peiris M (December 2013). "Middle East Respiratory Syndrome (MERS) coronavirus seroprevalence in domestic livestock in Saudi Arabia, 2010 to 2013". Euro Surveillance. 18 (50): 20659. doi:10.2807/1560-7917.es2013.18.50.20659. PMID 24342517 